\title{
New insights into the natural course and clinical relevance of Modic changes over 2 years following lumbar limited discectomy: analysis of prospective collected data
}

\author{
Richard Bostelmann $^{1}$ (D) $\cdot$ Athanasios Petridis ${ }^{1} \cdot$ Katinka Fischer $^{2} \cdot$ Peter Vajkoczy $^{3} \cdot$ Tamara Bostelmann $^{1}$. \\ Martin Barth ${ }^{4}$
}

Received: 1 November 2018 / Revised: 4 April 2019 / Accepted: 21 April 2019 / Published online: 4 May 2019

(c) The Author(s) 2019

\begin{abstract}
Purpose Few retrospective studies have addressed Modic changes (MC) following lumbar spine surgery, though it is usually assumed that MC increase in grade and incidence. To test this conventional wisdom, we investigated the natural course of MC following primary lumbar limited discectomy with two-year follow-up. In addition, a possible clinical relevance to those changes was assessed.

Methods The data of the control group (278 subjects) of a prospective randomized, controlled trial (RCT) were evaluated retrospectively.

Results We did not observe a simple increase in MC with regard to grade. There is variable activity observed in Type 2 (at 12 months) and in Type 1 (at 24 months). Conversion from one grade to another may occur and may be upward or downward. The incidence of MC increased slightly over time, as after surgery a decreasing percentage of the study group remained without MC over two years (1 year: 34\% (85/250); 2 years: 30\% (72/237)). Radiological parameters (rotation, translation, and spondylolisthesis) had no significant correlation to MC or MC subtypes. Lastly, we found that neither the different MC types nor their changes were correlated with clinical parameters (VAS back, VAS leg, ODI score) preoperatively or during follow-up.

Conclusion The pattern of Modic changes following lumbar limited discectomy is complex, not simply increasing. There is variable activity in MC Types 1 and 2 at the different time points of follow-up, and conversion from a higher grader to a lower one or vice versa is possible.
\end{abstract}

\section{Graphical abstract}

These slides can be retrieved under Electronic Supplementary Material.
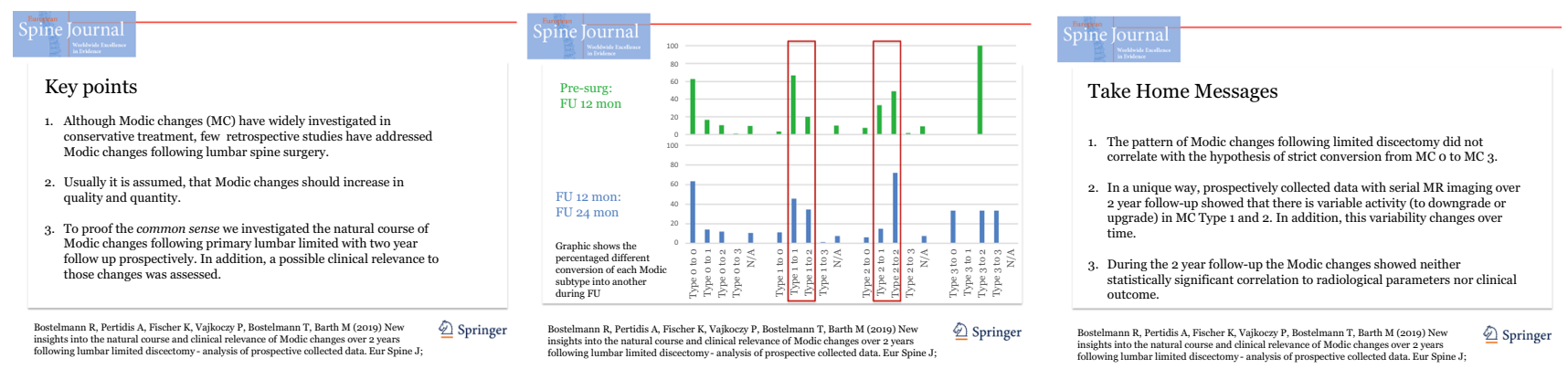

Electronic supplementary material The online version of this article (https://doi.org/10.1007/s00586-019-05988-1) contains supplementary material, which is available to authorized users.

Extended author information available on the last page of the article 
Keywords Spine $\cdot$ Low back pain $\cdot$ Modic change(s) $\cdot$ Degeneration $\cdot$ Discectomy $\cdot$ Magnetic resonance imaging

\section{Introduction}

Since the first description and classification of signal abnormalities of the vertebral endplate and subchondral bone marrow oedema in magnetic resonance tomography (MRI) of the lumbar spine, the interpretation and clinical correlation has been the subject of debate $[1,2]$. To date the understanding of Modic changes (MC) favours two main aetiologies: occult discitis [3] and autoimmune reaction [4]. These radiographic findings are understood as persistent stimuli, which presuppose structural disc damage [5]. In its physiological function, the disc is chronically exposed to forces and stresses. This may lead accumulatively to damage, which can also cause bone marrow oedema [6] or microfractures [7]. It has also been observed that biomechanical stress might contribute to the distribution of $\mathrm{MC}$ in the lumbar spine. In patients without specific pathology or surgery, MC were more common in the lowest two levels, especially L5-S1 [8]. Therefore, a biomechanical factor should also be considered in the pathogenesis of MC. In this context, an alteration of the biomechanical equilibrium is effected by iatrogenic damage of the disc. Correspondingly, a limited discectomy in the case of disrupted annulus fibrosus and herniated disc might alter the biomechanical forces directly on a microstructural level and predispose the adjacent vertebral to MC. Hence, we hypothesize that following discectomy the prevalence of MC should increase in both incidence and grade. And there is also little data on MC following lumbar discectomy [9-12]. To date there is no study available to evaluate the natural course of MC after a limited lumbar discectomy in a cohort of patients with prospectively collected data with a two-year follow-up and multiple imaging. This would allow real insight into these dynamic vertebral endplate changes, including any possible clinical relevance.

\section{Materials and methods}

\section{Study design}

This study describes outcomes of the control group only, from a multicenter, prospective randomized trial of subjects that underwent microscopic limited lumbar discectomy (only the herniated part of the disc was removed) and were followed for 2 years [13]. This study retrospectively reviewed the prospective data to analyse the natural course of $\mathrm{MC}$ in serial imaging. In addition, a possible clinical relevance of MC was assessed. The clinical trial (registered at ClinicalTrials.gov-NCT $01,283,438$ ) was approved by local ethics committees, and informed consent was obtained from all individual participants included in the study.

\section{Patient selection}

Two hundred seventy-eight patients (enrolled between December 2010 and October 2014) between 21 and 75 years of age with the presence of lumbar radiculopathy due to a primary, single-level disc herniation between $\mathrm{L} 1$ and $\mathrm{S} 1$, and failure of at least 6 weeks of nonsurgical treatment was eligible for the study. Exclusion criteria included prior indexlevel surgery, spondylolisthesis with $>25 \%$ slip, scoliosis of greater than 10 degrees (both angular and rotational), insulin-dependent diabetes, and contraindication for MRI. Further details were described in the study protocol published previously [14].

Baseline characteristics are presented in Table 1. Complete data sets including MRI images and evaluation of MC at the index level were available at 1 and 2 years for $89.9 \%$ (250/278) and 85.4\% (239/278) (Fig. 1). The mean volume of nucleus removal was $1.3 \mathrm{ml}$.

\section{Follow-up}

Clinical follow-up was done at 6 weeks, 3 months, 6 months, 1 year, and 2 years. MRI of the lumbar spine was performed preoperatively and at 1 and 2 years.

\section{MRI}

According to the imaging guidelines for the study, $\mathrm{T} 1$ and $\mathrm{T} 2$ images were required. Recommended axial slice thickness was 3-4 $\mathrm{mm}$ (sagittal $\leq 5 \mathrm{~mm}$ ), a gap equal or less than $1 \mathrm{~mm}$ (sagittal $\leq 1.5 \mathrm{~mm}$ ), and a maximum slice interval of $5 \mathrm{~mm}$ (sagittal $6.5 \mathrm{~mm}$ ).

X-ray According to the standard protocol, only true X-ray mode was used (no fluoro mode). For the lateral lumbar spine, $75-90 \mathrm{kVp}$ and $15-150 \mathrm{mAs}$ were preferred. Neutral-lateral, anteroposterior, and flexion/extension views were taken.

Magnetic resonance and conventional images were read by two board-certified radiologists at an independent imaging core laboratory (Intrinsic Imaging, LLC, Bolton, MA, USA) who were blinded to patient outcomes. Disagreement between raters was resolved with adjudication by a third radiologist. 
Table 1 Baseline characteristics of the patients

\begin{tabular}{lr}
\hline Characteristic & Study group $^{\mathrm{a}}(n=278)$ \\
\hline Age-year & $44.0(37.0-51.0)$ \\
Body mass index_-kg/m $^{2}$ a & $25.78(23.23-29.22)$ \\
Male sex-no. $(\%)$ & $171(62)$ \\
Visual analogue scale for leg pain $^{\mathrm{b}}$ & $81 \pm 15$ \\
Visual analogue scale for back pain $^{\mathrm{b}}$ & $56 \pm 31$ \\
Oswestry Disability Index score & $58 \pm 14$ \\
Smoking history-no. $(\%)$ & $175(63)$ \\
\hline
\end{tabular}

${ }^{a}$ Median values are given with the interquartile range in brackets

${ }^{\mathrm{b}}$ Scores on the visual analogue scale (VAS) range from 0 to 100 , with higher scores indicating more severe pain, reported as SD

${ }^{\mathrm{c}}$ Scores on the Oswestry Disability Index (ODI) range from 0 to 100, with higher scores indicating more severe disability, reported as SD

Fig. 1 Enrollment of Patients. Intent-to-treat (ITT) population consisted of 278 patients assigned to control group. Compliance with clinical follow-up and MRI Modic data sets at 1 year was $89.9 \%(250 / 278)$ and at 2 years was $85.4 \%(239 / 278)$

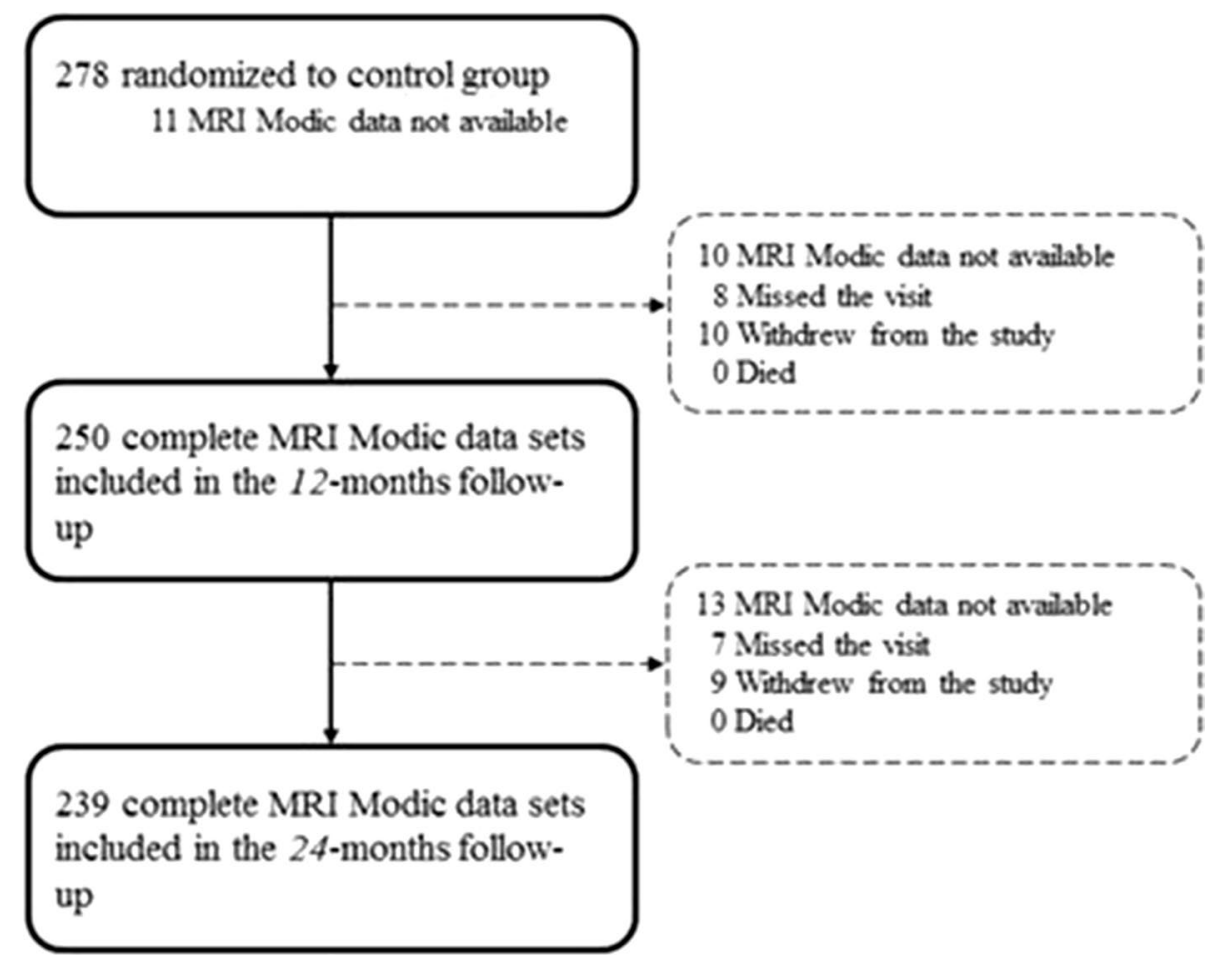

\section{Outcome measures}

\section{Radiological and MRI assessment}

Within three months prior to surgery, MRI with T1- and T2-weighted axial and sagittal images were performed. Neutral AP, lateral, and flexion-extension radiographs were performed within sixty days prior to surgery. Postoperative imaging was obtained at each annual follow-up (12 and 24 months). An independent radiological core laboratory analysed all radiological imaging. In this analysis, we concentrated on angular motion (index and adjacent), translational motion (index and adjacent), spondylolisthesis, and MC.
The Modic classification describes three types of different vertebral endplate changes on T1- and T2-weighted MRI $[2,15]$. To define the alterations of signal intensity changes at the index level during follow-up distinctly, there was the need for further subdividing each type into "new/increased", "not changed", or "decreased" signal intensity relative to baseline (Table 2).

\section{Clinical assessment}

Subjects' function and pain was assessed using the Oswestry Disability Index (ODI) [16] and visual analogue scales for back, right, and left leg pain using a $100-\mathrm{mm}$ scale. The 
Table 2 Description of sub-classification of Modic changes to assess the alterations in follow-up

\begin{tabular}{|c|c|c|c|}
\hline & Modic type & Subtype & Description of sub-classification \\
\hline 0 & 0 & None & $\begin{array}{l}\text { No oedematous reaction or vascular congestion induced in the adjacent bone marrow of the end- } \\
\text { plates }\end{array}$ \\
\hline 1 & 1 & New/increased & $\begin{array}{l}\text { Hypointense reaction and vascular congestion in the adjacent marrows on T1-weighted MR imag- } \\
\text { ing; hyperintense on T2-weighted images, new or increased relative to the previous time point }\end{array}$ \\
\hline 2 & 1 & Not changed & Modic Type 1 severity unchanged relative to the previous time point \\
\hline 3 & 1 & Decreased & $\begin{array}{l}\text { Modic Type } 1 \text { severity decreased relative to the previous time point, but not returned } \\
\text { to normal }\end{array}$ \\
\hline 4 & 2 & New/increased & $\begin{array}{l}\text { Bone marrow converted to a predominantly fatty marrow. Hyperintense on } \mathrm{T} 1 \text { and isointense to } \\
\text { hypointense on } \mathrm{T} 2 \text {. The exact signal intensity depends on the degree of } \mathrm{T} 2 \text { weighting }\end{array}$ \\
\hline 5 & 2 & Not changed & Modic Type 2 severity unchanged relative to the previous time point \\
\hline 6 & 2 & Decreased & $\begin{array}{l}\text { Modic Type } 2 \text { severity decreased relative to the previous time point, but not } \\
\text { returned to normal }\end{array}$ \\
\hline 7 & 3 & New/increased & $\begin{array}{l}\text { Dense sclerosis of the vertebral endplates. Reflected on MR imaging as hypointensity on both T1 } \\
\text { and T2-weighted images }\end{array}$ \\
\hline 8 & 3 & Not changed & Modic Type 3 severity unchanged relative to the previous time point \\
\hline 9 & 3 & Decreased & $\begin{array}{l}\text { Modic Type } 3 \text { severity decreased relative to the previous time point, but not } \\
\text { returned to normal }\end{array}$ \\
\hline
\end{tabular}

postoperative scores of the assessments were compared to baseline scores.

\section{Statistical analysis}

Baseline patient characteristics, including age, BMI, sex, smoking history, together with VAS (for back and leg pain), and ODI are presented as medians and interquartile ranges for continuous variables and percentages for categorical variables. In order to gain an impression of Modic changes over time (i.e. presurgery, after 12, and after 24 months), itemized data of Modic subtypes "new/ increased", "not changes", and "decreased" were pooled to Modic Types 1, 2 or 3, respectively, for reasons of clarity and comprehensibility. Results are represented as percentage values. Furthermore, an analysis of potential confounders, which could be a contributory cause of a specific Modic Type or Modic change over time, was carried out by the use of group comparisons between Modic Types and Modic changes over time regarding all collected patient characteristics. Initially, for each Modic Type the distribution of a potential confounder was visualized by boxplots for a continuous variable (e.g. age, BMI, size of annular defect, radiological findings) and by barplots for a categorical variable (e.g. gender, smoking,) with reference to the time point, i.e. presurgery, after 12, and after 24 months. Afterwards, possible significant differences between Modic Types regarding potential confounders were verified by using Student's $t$ test (in case of a two-group comparison) or ANOVA and, subsequently, Tukey's post hoc test (in case of more than two groups) for continuous data. In case of categorical data, Chi-square test was employed. Additionally, to analyse confounding variables regarding the conversion of Modic Types from one, e.g. after 12 months, to another, e.g. after 24 months, more precisely, group comparisons were employed. To subtype within one preoperative Modic Type, e.g. within group "preoperative Type 0", group comparisons between group "Type 0 after 12 months" and "Type 1 after 12 months" and so on, were made in the same way as described before. In case of non-normal distributed data and unbalanced groups, Mann-Whitney $\mathrm{U}$ test was employed. Statistical significance was set at $p<0.05$. Statistical analyses were performed using version 3.3.2 of the $R$ statistics package.

\section{Results}

\section{Modic changes}

Details are presented in Tables 3, 4, and 5. Results in per cent are based on the respective number with data for each follow-up examination.

Remarkably, there was a different pattern of transformation over time when analysing the MC Types and subtypes (Table 4). Preoperatively, MC Type 2 are observed to convert to Type 3 at one year in only $2 \%$ (2/121) of the cases, but a notably downward conversion to Type 1 and even to Type 0 at 1 and 2 years was observed [ 1 year FU: $33 \%$ (40/121) resp. 7\% (9/121); 2 years FU: 23\% (28/121) resp. 
Table 3 Modic changes of study population categorized into classical Modic types and defined subtypes (new/ increased, not changed, decreased) at presurgery (=baseline) and each follow-up

\begin{tabular}{llll}
\hline & $\begin{array}{l}\text { Presurgery }(=\text { base- } \\
\text { line }(n)\end{array}$ & FU 12 months $(n)$ & FU 24 months $(n)$ \\
\hline Modic Type & & & \\
Type 0 & $115[43 \%]$ & $85[35 \%]$ & $72[30 \%]$ \\
Type 1 & $30[11 \%]$ & $81[32 \%]$ & $65[27 \%]$ \\
New/ increased & N/A & 74 & 40 \\
Not changed & N/A & 6 & 23 \\
Decreased & N/A & 1 & 2 \\
Type 2 & $121[45 \%]$ & $81[32 \%]$ & $100[42 \%]$ \\
New/ increased & N/A & 20 & 26 \\
Not changed & N/A & 61 & 74 \\
Decreased & N/A & 0 & 0 \\
Type 3 & $1[1 \%]$ & $3[1 \%]$ & $2[1 \%]$ \\
New/ increased & N/A & 2 & 1 \\
Not changed & N/A & 1 & 1 \\
Decreased & N/A & 0 & 0 \\
Total available MRI Modic data & 267 [100\%] & $250[100 \%]$ & $239[100 \%]$ \\
Not available MRI Modic data & 11 & 28 & 39 \\
Sum & 278 & 278 & 278 \\
\hline
\end{tabular}

MRI Modic data not available due to different reasons: missing data from the core laboratory, withdrew from study, missed visit. Classical Modic types highlighted with light grey. The ratios are added in brackets [] relative to the total number of available MRI Modic data. The subcategories (new/increased, not changed, decreased) refer to the definitions for each group given in Table 2

\begin{tabular}{|c|c|c|c|c|c|c|}
\hline \multirow{2}{*}{$\begin{array}{l}\text { Conversion of } \mathrm{MC} \\
\text { from }\end{array}$} & \multicolumn{2}{|c|}{ Presurg: FU 12 m } & \multicolumn{2}{|c|}{ FU 12 m: FU 24 m } & \multicolumn{2}{|c|}{ Presurg: FU 24 m } \\
\hline & $(n)$ & $(\%)$ & $(n)$ & $(\%)$ & $(n)$ & $(\%)$ \\
\hline Type 0 & 115 & 100 & 85 & 100 & 115 & 100 \\
\hline to Type 0 & 72 & 62,6 & 54 & 63,5 & 56 & 48,7 \\
\hline to Type 1 & 19 & 16,5 & 12 & 14,1 & 20 & 17,4 \\
\hline to Type 2 & 12 & 10,4 & 10 & 11,8 & 23 & 20 \\
\hline to Type 3 & 1 & 0,9 & 0 & 0 & 0 & 0 \\
\hline N/A & 11 & 9,6 & 9 & 10,6 & 16 & 13,9 \\
\hline Type 1 & 30 & 100 & 81 & 100 & 30 & 100 \\
\hline to Type 0 & 1 & 3,3 & 9 & 11,1 & 1 & 3,3 \\
\hline to Type 1 & 20 & 66,7 & 37 & 45,7 & 16 & 53,3 \\
\hline to Type 2 & 6 & 20 & 28 & 34,6 & 8 & 26,7 \\
\hline to Type 3 & 0 & 0 & 1 & 1,2 & 0 & 0 \\
\hline N/A & 3 & 10 & 6 & 7,4 & 5 & 16,7 \\
\hline Type 2 & 121 & 100 & 81 & 100 & 121 & 100 \\
\hline to Type 0 & 9 & 7,4 & 5 & 6,1 & 12 & 9,9 \\
\hline to Type 1 & 40 & 33,1 & 12 & 14,8 & 28 & 23,1 \\
\hline to Type 2 & 59 & 48,8 & 58 & 71,6 & 64 & 52,9 \\
\hline to Type 3 & 2 & 1,7 & 0 & 0 & 2 & 1,7 \\
\hline N/A & 11 & 9,1 & 6 & 7,4 & 15 & 12,4 \\
\hline Type 3 & 1 & 100 & 3 & 100 & 1 & 100 \\
\hline to Type 0 & 0 & 0 & 1 & 33,3 & 0 & 0 \\
\hline to Type 1 & 0 & 0 & 0 & 0 & 0 & 0 \\
\hline to Type 2 & 1 & 100 & 1 & 33,3 & 1 & 100 \\
\hline to Type 3 & 0 & 0 & 1 & 33,3 & 0 & 0 \\
\hline N/A & 0 & 0 & 0 & 0 & 0 & 0 \\
\hline
\end{tabular}

Table 4 Conversion of Modic changes categorized into Types (0-3) between each follow-up and presurgical (=baseline) situation. Percentage values based on the respective population in each categorized subtype. Not categorized images are not shown 
Table 5 Visual analogue scale for back pain and leg pain and ODI score of investigated population and their statistically significance

\begin{tabular}{cllllll}
\hline & Presurgery & 12 -mo FU & 24 -mo FU & $\begin{array}{l}p \text { value } \\
\text { (pre/12 } \\
\text { mons) }\end{array}$ & $\begin{array}{l}p \text { value } \\
\text { (pre/24 } \\
\text { mons })\end{array}$ & $\begin{array}{l}p \text { value } \\
(12 / 24 \\
\text { mons })\end{array}$ \\
\hline VAS for back pain & $56 \pm 31$ & $21 \pm 24$ & $19 \pm 24$ & 0.000 & 0.000 & 0.2451 \\
Type 0 & $53 \pm 32$ & $20 \pm 25$ & $17 \pm 22$ & 0.000 & 0.000 & 0.9944 \\
Type 1 & $67 \pm 27$ & $24 \pm 26$ & $24 \pm 28$ & 0.000 & 0.000 & 0.5581 \\
Type 2 & $55 \pm 32$ & $19 \pm 22$ & $15 \pm 21$ & 0.000 & 0.000 & 0.0375 \\
Type 3 & $92 \pm 0$ & $21 \pm 29$ & $43 \pm 61$ & N/A & N/A & N/A \\
VAS for leg pain & $81 \pm 15$ & $14 \pm 21$ & $14 \pm 21$ & 0.000 & 0.000 & 0.6313 \\
Type 0 & $80 \pm 15$ & $14 \pm 20$ & $13 \pm 18$ & 0.000 & 0.000 & 0.9673 \\
Type 1 & $81 \pm 14$ & $13 \pm 22$ & $14 \pm 20$ & 0.000 & 0.000 & 0.7544 \\
Type 2 & $81 \pm 15$ & $14 \pm 23$ & $13 \pm 22$ & 0.000 & 0.000 & 0.8566 \\
Type 3 & $70 \pm 0$ & $11 \pm 15$ & $42 \pm 59$ & N/A & N/A & N/A \\
ODI & $58 \pm 14$ & $14 \pm 15$ & $14 \pm 15$ & 0.000 & 0.000 & 0.4547 \\
Type 0 & $57 \pm 13$ & $14 \pm 17$ & $13 \pm 14$ & 0.000 & 0.000 & 0.5880 \\
Type 1 & $54 \pm 11$ & $14 \pm 16$ & $16 \pm 15$ & 0.000 & 0.000 & 0.4007 \\
Type 2 & $59 \pm 15$ & $13 \pm 14$ & $12 \pm 13$ & 0.000 & 0.000 & 0.2982 \\
Type 3 & $72 \pm 0$ & $10 \pm 14$ & $46 \pm 40$ & N/A & N/A & N/A \\
\hline
\end{tabular}

Scores on the visual analogue scale (VAS) range from 0 to 100, with higher scores indicating more severe pain. Scores on the Oswestry Disability Index (ODI) range from 0 to 100, with higher scores indicating more severe disability. Pre $=$ presurgery, mons $=$ months. Significance was set at $>0.5$. Although statistical significance for MC Type 3 could be shown, these results were not considered as relevant due to the small sample size of these groups (therefore N/A)
$10 \%(12 / 121)]$. The first year following surgery seems to be the most active phase in Type 2, whereas Type 1 changes seem to have the most active phase of change between the 12 and 24 month follow-ups (35\%, 28 out of 81 convert to
Type 2). Preoperatively MC Type 0 has a constant pattern of change to Type 1 ( 1 year FU: 17\%, 2 years FU: 14\%) and Type 2 (1 year FU: 10\%, 2 years FU: 12\%). Type 3 seems to be a less active situation of bone marrow changes.
Fig. 2 The percentage conversion of each Modic Type (0-3) and subtype (new/increased, not changed, decreased) into another at 12 months relative to presurgery (=baseline)

\section{Pre-surg: FU $12 \mathrm{~m}$}

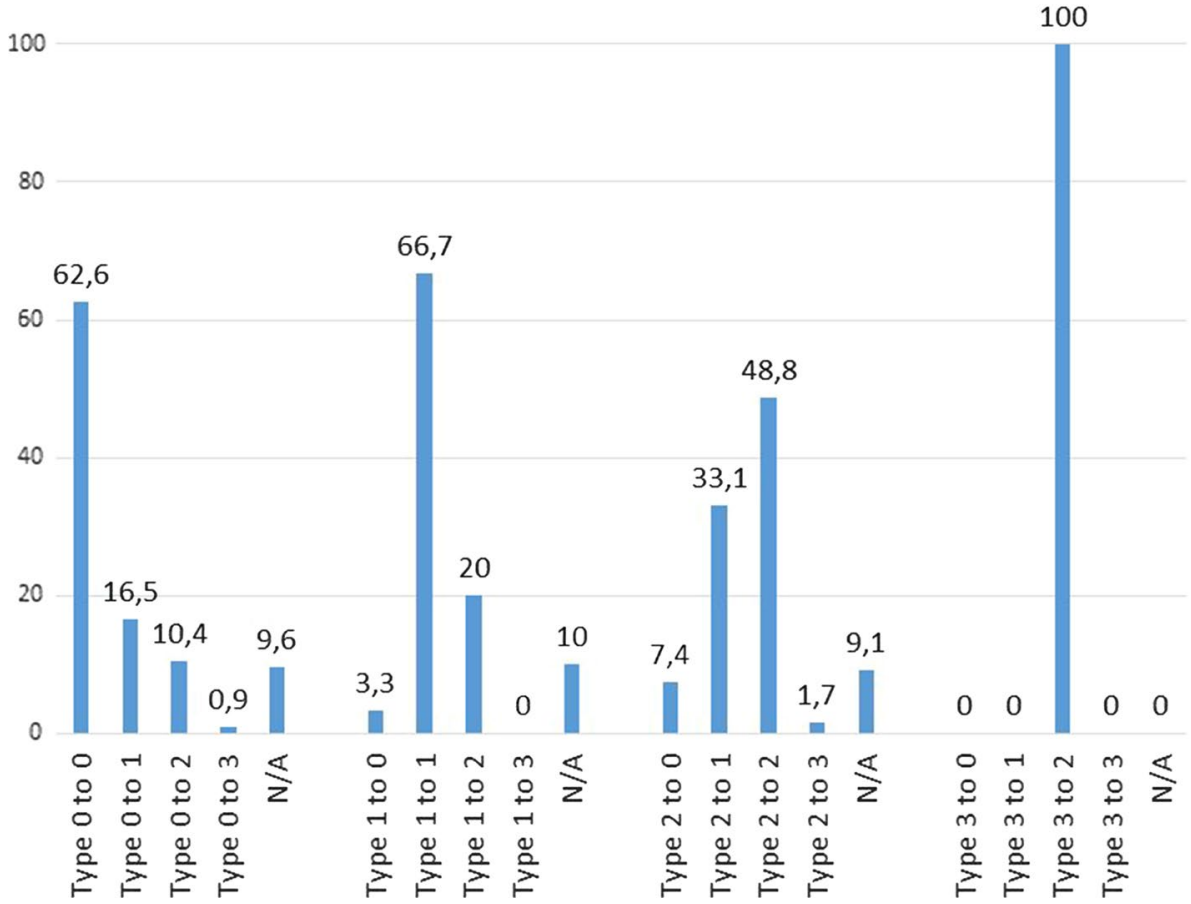


Fig. 3 The percentage conversion of each Modic Type (0-3) and subtype (new/increased, not changed, decreased) into another at 24 months relative to 12 months

Fig. 4 The percentage conversion of each Modic Type (0-3) and subtype (new/increased, not changed, decreased) into another at 24 months relative to presurgery
FU 12 m : FU 24 m

100

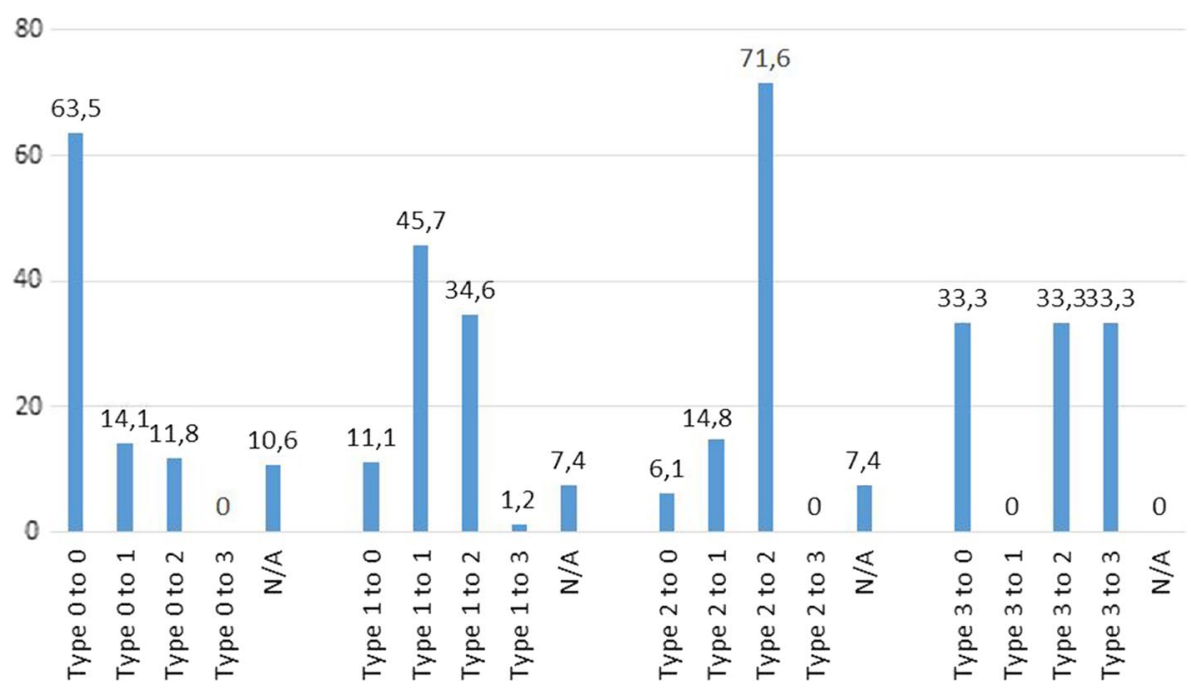

Pre-surg : FU 24 m

100

100

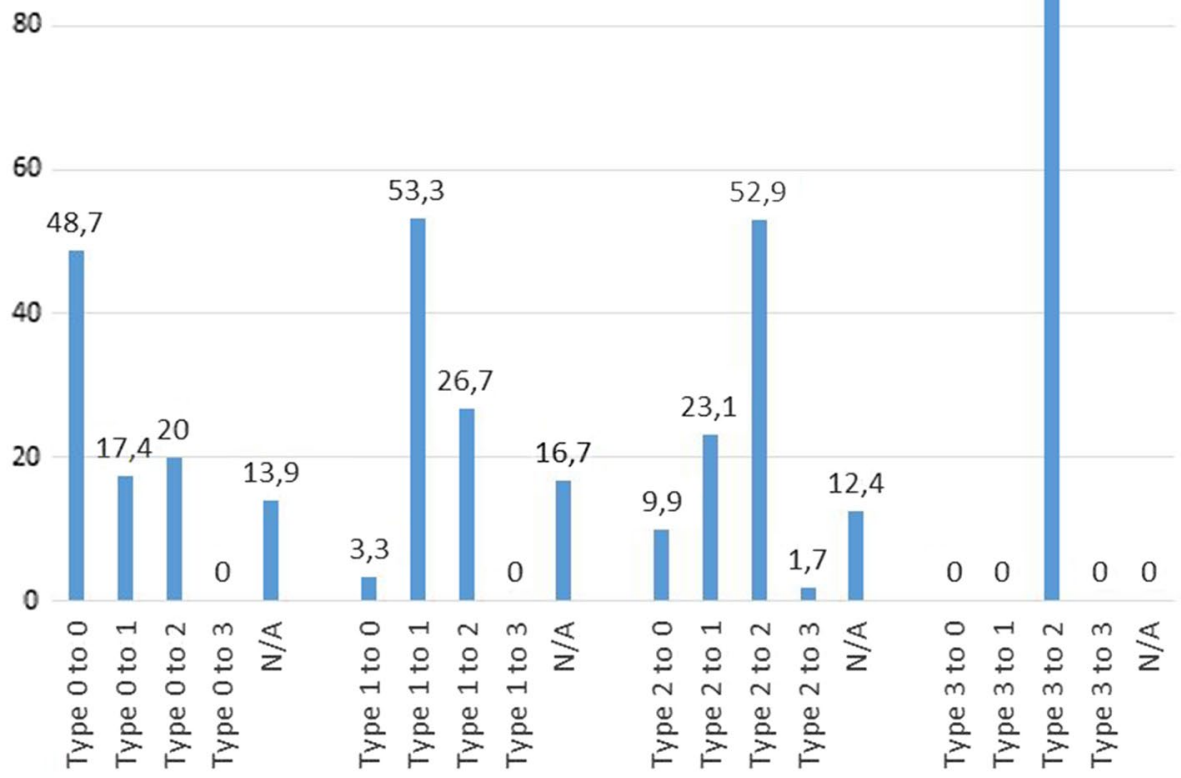

The percentage changes of MC type and subtype are shown in Table 4. The majority of MC remain in their subtype between presurgery and 12 months (62.6\% Type 0 , $66.7 \%$ Type $1,48.8 \%$ Type 2 ) and between 12 and 24 months (63.5\% Type $0,45.7 \%$ Type $1,71.6 \%$ Type 2 ). Between presurgery and 12 months, it was observed that $33.1 \%$ Type 2 converted into Type 1 (at 12 months/24 months $14.8 \%$ ) and that $20.0 \%$ Type 1 converted into Type 2 (at 12 months/24 months $34.6 \%$ ). Comparing MC between presurgery and 24 months, nearly 50\% remain unchanged 
(48.7\% Type 0, 53.3\% Type 1, 52.9\% Type 2). Further details are shown in Figs. 2, 3 and 4.

\section{Relationship of MC to clinical outcomes}

Subjects achieved a highly significant $(p<0.0001)$ improvement in back pain, leg pain, and ODI score, at the regular 12 -month visit. This clinically meaningful improvement was stable at 24 months. Regarding the sub-analysis of Modic types, no significant differences were found concerning these outcome parameters (Table 5). Even though 70\% of subjects had recurrent herniations identified on imaging regardless of symptoms at 24 months, only 45 patients ( 37 at the index level) needed further surgical treatment.

For sensitivity assurance, patients re-operated for symptomatic reherniation within 2 years were excluded from the analysis. This did not change the reported significances.

\section{Radiological parameters and MC}

During the 2-year follow-up period, no statistically significant difference could be observed between rotation, translation, and spondylolisthesis at the index level and these radiological characteristics at baseline. Moreover, no correlations to MC Types 1, 2, or 3 nor to lack of MC were observed.

Rotational motion was $-4.5^{\circ}\left(-1.4^{\circ}\right.$ to $\left.-7.1^{\circ}\right)$ at baseline, $-4.3^{\circ}\left(-1.4^{\circ}\right.$ to $\left.-6.6^{\circ}\right)$ at 12 months, and $-4.3^{\circ}\left(-1.3^{\circ}\right.$ to $\left.-6.9^{\circ}\right)$ at 24 months. Translational motion was $0.3 \mathrm{~mm}(1.1$ to -0.7$)$ at baseline, $0.5 \mathrm{~mm}(1.2$ to -0.4$)$ at 12 months, and $0.2 \mathrm{~mm}(1$ to -0.6$)$ at 24 months. Spondylolisthesis was $-1.6 \mathrm{~mm}(-0.8$ to -2.6$)$ at baseline, $-1.5 \mathrm{~mm}(-0.9$ to -2.6$)$ at 12 months, and $-1.3 \mathrm{~mm}(-0.6$ to -2.2$)$ at 24 months.

\section{Further parameters and MC}

No statistically significant correlations could be observed between MC and age, sex, BMI, or smoking status. Insulindependent diabetes was excluded from the study.

\section{Discussion}

To date this is the largest retrospective analysis of prospectively collected data to evaluate the natural course of Modic changes following a limited discectomy in lumbar spine with serial control visits up to two years. Much to our surprise, we did not observe a simple evolution of MC with increasing frequency and grade of changes. The pattern is much more complex. The majority of Modic changes remain in their subtype comparing presurgery to 12 months and 12 months to 24 months. In addition, there is variable activity observed in Type 2 (e.g. downgrading at 12 months) and in Type 1 (e.g. upgrading between 12 and 24 months). Conversion from one grade to another may occur and may be upward or downward (not only MC Type $0-1$, MC Type $1-2$, or MC Type 2-3), whereas a change to Type 3 occurs less frequently. Comparing the changes at 24 months relative to presurgery, the dynamic variances of MC following surgery are less obvious. The impression that $\mathrm{MC}$ only progress following disc surgery reported elsewhere $[10,17]$ might be due to the lack of serial imaging in a dynamic event like Modic changes. The phenomenon of downgrading that we observed was also reported in the results of a longitudinal cohort study with conservatively treated lumbar disc herniation with $1.2 \%$ (2/166) for Type 1 and $6.6 \%(11 / 166)$ for Type 2 after 14 months [9]. Of note, a downward conversion of 33\% of MC Type 2 to Types 1 and 0 was observed in our prospective study group at two years. Therefore, the assumption of a strict conversion from MC 0 to MC 3 should be reconsidered in the case of surgery for disc herniation. In addition, this is supported by the observation of a nonlinear evolution in MC types in a retrospective analysis of 358 patients with a medical history of discectomy [18].

In our investigation, we observed that $45 \%$ of surgical candidates for lumbar discectomy (mean age 44.0 years) had no MC in their imaging before surgery. Similar observations were reported in an observational study with a prevalence of no MC in $44.4 \%$ (249 individuals) with a mean age of 47.7 years [19]. Furthermore, we found MC most frequently in the lower lumbar region (adjacent to L4/5 and L5/S1) which has also been described elsewhere [8, 19]. Therefore, despite the limitations of clinical study conditions, distribution and prevalence of $\mathrm{MC}$ are close to what has previously been described in the literature $[8,19,20]$.

Of note, there are different characteristics of MRI findings in the human lumbar spine besides MC, for instance disc displacement and endplate lesions. However, their prevalence is questionable. Some found endplate defects in two-thirds of their investigated cohort (108 patients) and showed a significance with disc degeneration scores, $\mathrm{MC}$, and posterior disc displacement [21]. Others detected no endplate lesions in the whole lumbar spine in nearly two-thirds of 996 low back pain patients investigated with MRI [22]. Moreover, they observed a clear association between the presence of endplate defects and disc degeneration, but not for MC [22]. We concentrated on Modic changes following surgery for single-level disc herniation with unresponsiveness to conservative treatment. We did not evaluate different reasons for lumbar back pain as $\mathrm{MC}$ at others levels. But, main reasons such as instability, discitis, severe degeneration, and tumour were ruled out per study protocol [14].

Interestingly, prospective MRI investigation in a Finish population provided particular insights in unspecific chronic low back pain patients [23]. Baseline MRI with MC Type 1 was compared to follow-up. They found also up- and downgrading of MC in their highly selected 54 subjects. 
They did not present a distinct pattern, but they hypothesized a link between endplate lesions and subchondral signal abnormalities. Kerttula et al. reported in 54 subjects after 11-18 months of follow-up of constant MC Type 1 in $9 \%$, increased in $40 \%$, and decreased in $45 \%$ [24]. This is only partly comparable to our findings at 12 months followup showing two-thirds of MC Type 1 had no conversion to another type. Of these, the majority $(91 \%)$ were new or increased. These differences may be mostly due to the different study population and study protocol (period for followup MRI, female preponderance, all disc levels included, no surgical candidates, and low back pain patients). However, it highlights the fact that also in low back pain patients a distinct pattern of change in MC Type 1 (increase, disappear, or change into another Type-MC Type 2) exists, even though their proportional prevalence is not similar to our findings. The similarities of the findings are supported by the consideration that $\mathrm{MC}$ seems to be a combination of endplate or disc injury, inflammatory mediators, and lowgrade infection [25].

In our study population, we observed more MC following surgery than before surgery. MC subtypes had different patterns over time. Whereas MC Type 1 markedly increased by sevenfold (81/30) at the twelve months follow-up, MC Type 2 was reduced more than fivefold (81/121), and at 24 months the pattern was inverse as Type 1 decreased (20\%) and Type 2 increased (19\%). This leads us to the interpretation that the reaction to mechanical stress is different and long lasting. This is underlined by the results of a minor cohort of 41 subjects, who received a surgical disc prolapse removal [10]. After a median follow-up of 41 months, Rahme et al. found an increase of $81 \%$ in $\mathrm{MC}$ at the operated level (46.3\% to $78 \%$, being Type 2 lesions in 26 out of 32 cases). A conversion to Type 0 was not observed. In contrast, this conversion from Type 1 to Type 0 was observed after posterior screwrod osteosynthesis and posterolateral arthrodesis. When disc degeneration after discectomy was the reason for fusion procedure, a recovery to no MC was observed in 57\% [26]. Furthermore, an animal model confirmed that chronic compression led to significantly increased vertebral bone marrow oedema in mice [6]. In addition, a biomechanical testing on 27 human cadaveric lumbar spines found that in the presence of MC (or degenerative disc) the strength and stiffness of the bony endplate decreased greatly, by up to $46 \%$ [27]. Therefore, it should be evident that biomechanical stress leads to MC in the case of disc herniation, considering that loss of nucleus material (by herniation or more violently by surgery) reduces intradiscal pressure [28] and subsequently alters the pressure load distribution over the disc [29]. Due to their physical nature, these alterations are therefore likely occurring before they are evident on imaging evident below an imaging level. Moreover, a severe spinal imbalance that would further influence segmental function was ruled out by the exclusion criteria. Additionally, postoperative radiological assessment showed no segmental hypermobility after surgical procedure.

\section{Limitation}

First, due to the nature of this study (control arm of an RCT) we focused on a selected group of subjects. We therefore can only extrapolate our results to patients with symptomatic disc herniation. There are other aetiologies discussed in the literature, which might be linked with MC such as chronic low back pain. Second, this study focused on single-level pathology in the context of disc herniation. We did not consider further possible reasons for low back pain originating from different levels than the treated one or generated by endplate lesions. Third, presence of low virulent anaerobic microorganisms in the disc samples was not investigated. However, all subjects were immunocompetent and treaded conservatively at least for 6 weeks, which did not rule out treatment with steroids. Finally, longer patient follow-up is required to draw more definite conclusions regarding Modic changes.

\section{Conclusion}

In longitudinal MRI investigation, the pattern of Modic changes following lumbar limited discectomy is complex and not simply increasing. There is variable activity in MC Types 1 and 2 at the different time points of follow-up. Downgrading or upgrading of observed MC is possible. In the first year, the majority of MC Type 1 stay stable but onethird of MC Type 2 is converting (upgrading). In the second year, this changes to the opposite, as the majority of MC Type 2 stay stable but one-third of MC Type 1 is converting (downgrading). During the 2-year follow-up, the MC did not show statistically significant correlation to either radiological parameters or clinical outcome. Interpretation of these results in the context of chronic low back pain should be done with caution.

Acknowledgments We thank Oscar Yeh of Intrinsic Therapeutics for his assistance in data analysis and the fruitful discussions to guarantee the data's integrity.

\section{Compliance with ethical standards}

Conflict of interest No funds were received in support of this work. No benefits in any form have been or will be received from a commercial party related directly or indirectly to the subject of this manuscript.

Open Access This article is distributed under the terms of the Creative Commons Attribution 4.0 International License (http://creativeco 
mmons.org/licenses/by/4.0/), which permits unrestricted use, distribution, and reproduction in any medium, provided you give appropriate credit to the original author(s) and the source, provide a link to the Creative Commons license, and indicate if changes were made.

\section{References}

1. de Roos A, Kressel H, Spritzer C, Dalinka M (1987) MR imaging of marrow changes adjacent to end plates in degenerative lumbar disk disease. AJR Am J Roentgenol 149:531-534. https://doi. org/10.2214/ajr.149.3.531

2. Modic MT, Steinberg PM, Ross JS, Masaryk TJ, Carter JR (1988) Degenerative disk disease: assessment of changes in vertebral body marrow with MR imaging. Radiology 166:193-199. https ://doi.org/10.1148/radiology.166.1.3336678

3. Albert HB, Kjaer P, Jensen TS, Sorensen JS, Bendix T, Manniche $\mathrm{C}$ (2008) Modic changes, possible causes and relation to low back pain. Med Hypotheses 70:361-368. https://doi.org/10.1016/j. mehy.2007.05.014

4. Ma XL, Ma JX, Wang T, Tian P, Han C (2011) Possible role of autoimmune reaction in Modic Type I changes. Med Hypotheses 76:692-694. https://doi.org/10.1016/j.mehy.2011.01.035

5. Dudli S, Fields AJ, Samartzis D, Karppinen J, Lotz JC (2016) Pathobiology of Modic changes. Eur Spine J 25:3723-3734. https ://doi.org/10.1007/s00586-016-4459-7

6. Papuga MO, Proulx ST, Kwok E, You Z, Rubery PT, Dougherty PE, Hilton MJ, Awad HA, Schwarz EM (2010) Chronic axial compression of the mouse tail segment induces MRI bone marrow edema changes that correlate with increased marrow vasculature and cellularity. J Orthop Res 28:1220-1228. https://doi. org/10.1002/jor.21103

7. Malinin T, Brown MD (2007) Changes in vertebral bodies adjacent to acutely narrowed intervertebral discs: observations in baboons. Spine 32:E603-E607. https://doi.org/10.1097/ BRS.0b013e31815574e7

8. Huang ZY, Xu HC, Lei T, Li QL, Wu AM, Ni WF (2016) The location of Modic changes in the lumbar spine: a meta-analysis. Eur Spine J 25:3746-3759. https://doi.org/10.1007/s0058 6-016-4456-x

9. Albert HB, Manniche C (2007) Modic changes following lumbar disc herniation. Eur Spine J 16:977-982. https://doi.org/10.1007/ s00586-007-0336-8

10. Rahme R, Moussa R, Bou-Nassif R, Maarrawi J, Rizk T, Nohra G, Samaha E, Okais N (2010) What happens to Modic changes following lumbar discectomy? Analysis of a cohort of 41 patients with a 3- to 5-year follow-up period. J Neurosurg Spine 13:562567. https://doi.org/10.3171/2010.5.SPINE09818

11. Barth M, Weiss C, Bouma GJ, Bostelmann R, Kursumovic A, Fandino J, Thome C (2018) Endplate changes after lumbar discectomy with and without implantation of an annular closure device. Acta Neurochir (Wien) 160:855-862. https://doi.org/10.1007/ s00701-017-3463-y

12. Hutton MJ, Bayer JH, Powell JM (2011) Modic vertebral body changes: the natural history as assessed by consecutive magnetic resonance imaging. Spine 36:2304-2307. https://doi.org/10.1097/ BRS.0b013e31821604b6

13. Thome C, Klassen PD, Bouma GJ, Kursumovic A, Fandino J, Barth M, Arts M, van den Brink W, Bostelmann R, Hegewald A, Heidecke V, Vajkoczy P, Frohlich S, Wolfs J, Assaker R, Van de Kelft E, Kohler HP, Jadik S, Eustacchio S, Hes R, Martens F, Annular Closure RCTSG (2018) Annular closure in lumbar microdiscectomy for prevention of reherniation: a randomized clinical trial. Spine J. https://doi.org/10.1016/j.spinee.2018.05.003

14. Klassen PDHR, Bouma GL, Eustacchio S, Barth M, Kursumovic A, Jadik S, Heidecke V, Bostelmann R, Thomé C, Vajkoczy P, Köhler HP, Fandino J, Assaker R, Kelft E, Fröhlich S, Brink W, Wolfs J, Arts M, Martens F (2016) A multicenter, prospective, randomized study protocol to demonstrate the superiority of a bone-anchored prosthesis for anular closure used in conjunction with limited discectomy to limited discectomy alone for primary lumbar disc herniation. Int J Clin Trials 3:120-131. https://doi. org/10.18203/2349-3259.ijct20162794

15. Modic MT, Masaryk TJ, Ross JS, Carter JR (1988) Imaging of degenerative disk disease. Radiology 168:177-186. https://doi. org/10.1148/radiology.168.1.3289089

16. Fairbank JC, Pynsent PB (2000) The Oswestry Disability Index. Spine 25:2940-2952 (discussion 2952)

17. Barth M, Diepers M, Weiss C, Thome C (2008) Two-year outcome after lumbar microdiscectomy versus microscopic sequestrectomy: part 2: radiographic evaluation and correlation with clinical outcome. Spine 33:273-279. https://doi.org/10.1097/ BRS.0b013e31816201a6

18. Mostofi K, Moghaddam BG, Peyravi M (2018) Late appearance of low back pain relating to Modic change after lumbar discectomy. J Craniovertebr Junction Spine 9:93-95. https://doi.org/10.4103/ jcvjs.JCVJS_25_18

19. Wang Y, Videman T, Battie MC (2012) Modic changes: prevalence, distribution patterns, and association with age in white men. Spine J 12:411-416. https://doi.org/10.1016/j.spinee.2012.03.026

20. Kuisma M, Karppinen J, Niinimaki J, Ojala R, Haapea M, Heliovaara M, Korpelainen R, Taimela S, Natri A, Tervonen O (2007) Modic changes in endplates of lumbar vertebral bodies: prevalence and association with low back and sciatic pain among middle-aged male workers. Spine 32:1116-1122. https://doi. org/10.1097/01.brs.0000261561.12944.ff

21. Zehra U, Cheung JPY, Bow C, Lu W, Samartzis D (2018) Multidimensional vertebral endplate defects are associated with disc degeneration, Modic changes, facet joint abnormalities and pain. J Orthop Res. https://doi.org/10.1002/jor.24195

22. Brayda-Bruno M, Albano D, Cannella G, Galbusera F, Zerbi A (2018) Endplate lesions in the lumbar spine: a novel MRIbased classification scheme and epidemiology in low back pain patients. Eur Spine J 27:2854-2861. https://doi.org/10.1007/s0058 6-018-5787-6

23. Luoma K, Vehmas T, Kerttula L, Gronblad M, Rinne E (2016) Chronic low back pain in relation to Modic changes, bony endplate lesions, and disc degeneration in a prospective MRI study. Eur Spine J 25:2873-2881. https://doi.org/10.1007/s0058 6-016-4715-x

24. Kerttula L, Luoma K, Vehmas T, Gronblad M, Kaapa E (2012) Modic type I change may predict rapid progressive, deforming disc degeneration: a prospective 1-year follow-up study. Eur Spine J 21:1135-1142. https://doi.org/10.1007/s00586-012-2147-9

25. Crockett MT, Kelly BS, van Baarsel S, Kavanagh EC (2017) Modic Type 1 Vertebral Endplate Changes: Injury, Inflammation, or Infection? AJR Am J Roentgenol 209:167-170. https:// doi.org/10.2214/AJR.16.17403

26. Vital JM, Gille O, Pointillart V, Pedram M, Bacon P, Razanabola F, Schaelderle C, Azzouz S (2003) Course of Modic 1 six months after lumbar posterior osteosynthesis. Spine (Phila Pa 1976) 28:715-720. https://doi.org/10.1097/01.BRS.0000051924.39568 .31(discussion 721)

27. Liu J, Hao L, Suyou L, Shan Z, Maiwulanjiang M, Li S, Wang C, Fan S, Zhao F (2016) Biomechanical properties of lumbar endplates and their correlation with MRI findings of lumbar 
degeneration. J Biomech 49:586-593. https://doi.org/10.1016/j. jbiomech.2016.01.019

28. Bostelmann R, Steiger HJ, Cornelius JF (2017) Effect of Annular Defects on Intradiscal Pressures in the Lumbar Spine: An in Vitro Biomechanical Study of Diskectomy and Annular Repair. J Neurol Surg A Cent Eur Neurosurg 78:46-52. https://doi. org/10.1055/s-0035-1570344

29. Steffen T, Baramki HG, Rubin R, Antoniou J, Aebi M (1998)

Lumbar intradiscal pressure measured in the anterior and posterolateral annular regions during asymmetrical loading. Clin Biomech (Bristol, Avon) 13:495-505

Publisher's Note Springer Nature remains neutral with regard to jurisdictional claims in published maps and institutional affiliations.

\section{Affiliations}

\section{Richard Bostelmann ${ }^{1}$ (1) Athanasios Petridis ${ }^{1} \cdot$ Katinka Fischer $^{2} \cdot$ Peter Vajkoczy $^{3} \cdot$ Tamara Bostelmann $^{1}$. Martin Barth ${ }^{4}$}

$\triangle$ Richard Bostelmann

richard.bostelmann@med.uni-duesseldorf.de

1 Department of Neurosurgery, Medical Faculty, University Hospital Duesseldorf, Moorenstrasse 5, 40225 Duesseldorf, Germany

2 Mathematical Institute, Heinrich Heine University, Duesseldorf, Germany
3 Department of Neurosurgery, Charité Universitätsmedizin, Berlin, Germany

4 Department of Neurosurgery, Klinikum Frankfurt Höchst, Frankfurt, Germany 